\title{
Dark Stars: Död och Återuppståndelse
}

\author{
Douglas Spolyar ${ }^{* 1}$, Katherine Freese ${ }^{2}$, Paolo Gondolo ${ }^{3}$, Anthony Aguirre ${ }^{1}$, Peter \\ Bodenheimer $^{1}$, and Naoki Yoshida ${ }^{4}$ \\ ${ }^{1}$ University of California, Santa Cruz, ${ }^{2}$ University of Michigan, ${ }^{3}$ University of Utah, ${ }^{4}$ University \\ of Tokyo \\ E-mail: dspolyar@physics.ucsc.edu, ktfreese@umich.edu, \\ paolo@physics.utah.edu, aguirre@scipp.ucsc.edu, \\ peter@ucolick.org,nyoshidala.phys.nagoya-u-ac.jp
}

\begin{abstract}
The first phase of stellar evolution in the history of the universe may be Dark Stars, powered by dark matter heating rather than by fusion. Weakly interacting massive particles, which are their own antiparticles, can annihilate and provide an important heat source for the first stars in the universe. This and the previous contribution present the story of Dark Stars. In this second part, we describe the structure of Dark Stars and predict that they are very massive $\left(\sim 800 M_{\odot}\right)$, cool $(6000 \mathrm{~K})$, bright $\left(\sim 10^{6} L_{\odot}\right)$, long-lived ( $\sim 10^{6}$ years), and probable precursors to (otherwise unexplained) supermassive black holes. Later, once the initial dark matter fuel runs out and fusion sets in, dark matter annihilation can predominate again if the scattering cross section is strong enough, so that a Dark Star is born again.
\end{abstract}

Identification of dark matter 2008

August 18-22, 2008

Stockholm, Sweden

\footnotetext{
* Speaker.
} 


\section{Building up the Mass}

Recently, we have found the stellar structure of the dark stars (hereafter DS) ([1]). Though they form with the properties mentioned in the accompanying contribution ([2]), they continue to accrete mass from the surrounding medium. In ([1]) we build up the DS mass as it grows from $\sim 1 M_{\odot}$ to $\sim 1000 M_{\odot}$. As the mass increases, the DS contracts and the DM density increases until the DM heating matches its radiated luminosity. We find polytropic solutions for dark stars in hydrostatic and thermal equilibrium. We start with a few $M_{\odot}$ DS and find an equilibrium solution. Then we build up the DS by accreting $1 M_{\odot}$ at a time with an accretion rate of $2 \times 10^{-3} M_{\odot} / \mathrm{yr}$, always finding equilibrium solutions. We find that initially the DS are in convective equilibrium; from $(100-400) M_{\odot}$ there is a transition to radiative; and heavier DS are radiative. As the DS grows, it pulls in more DM, which then annihilates. We continue this process until the DM fuel runs out at $M_{D S} \sim 800 M_{\odot}$ (for $100 \mathrm{GeV}$ WIMPs). Figure 3 shows the stellar structure. One can see "the power of darkness:" although the DM constitutes a tiny fraction $\left(<10^{-3}\right)$ of the mass of the DS, it can power the star. The reason is that WIMP annihilation is a very efficient power source: $2 / 3$ of the initial energy of the WIMPs is converted into useful energy for the star, whereas only $1 \%$ of baryonic rest mass energy is useful to a star via fusion.

\section{Results and Predictions}

Our final result ([1]), is very large first stars. For example, for $100 \mathrm{GeV}$ WIMPs, the first stars have $M_{D S}=800 M_{\odot}$. Once the DM fuel runs out inside the DS, the star contracts until the central temperature reaches $10^{8} \mathrm{~K}$ and fusion sets in. A possible end result of stellar evolution will be large black holes. The Pair Instability SN ([3]) that would be produced from 140-260 $M_{\odot}$ stars (and whose chemical imprint is not seen) would not be as abundant. Indeed this process may help

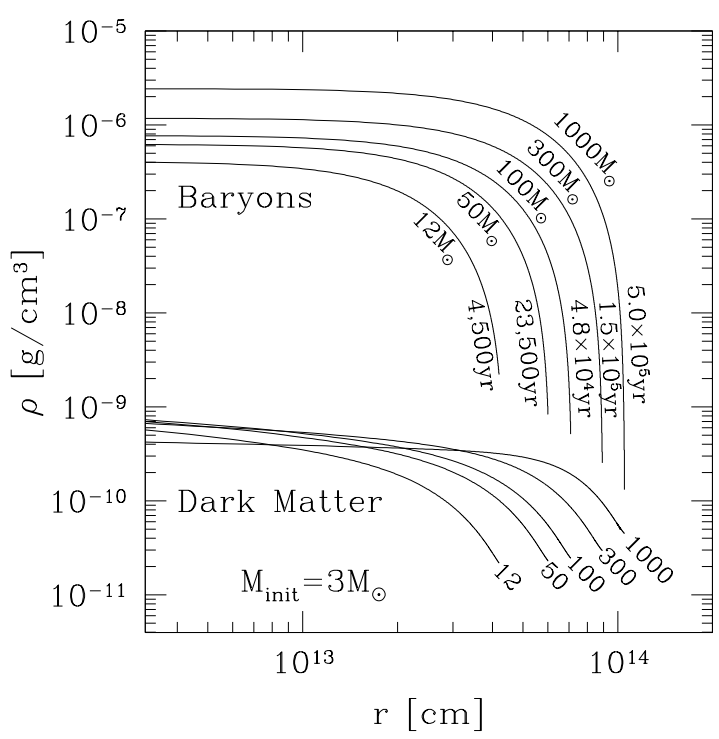

Figure 1: Evolution of a dark $\operatorname{star}(n=1.5)$ as mass is accreted onto the initial protostellar core of $3 \mathrm{M}_{\odot}$. The set of upper (lower) curves correspond to the baryonic (DM) density profile at different masses and times. Note that DM constitutes $<10^{-3}$ of the mass of the DS. 
to explain the supermassive black holes that have been found at high redshift $\left(10^{9} M_{\odot} \mathrm{BH}\right.$ at $\left.\mathrm{z}=6\right)$ and are, as yet, unexplained ([4]; [5]).

The lifetime of this new DM powered phase of stellar evolution, prior to the onset of fusion, is $\sim 10^{6}$ years. The stars are very bright, $\sim 10^{6} L_{\odot}$, and relatively cool, $(6000-10,000) \mathrm{K}$ (as opposed to standard Pop III stars whose surface temperatures exceed 30,000K). Reionization during this period is likely to be slowed down, as these stars can heat the surroundings but not ionize them. One can thus hope to find DS and differentiate them from standard Pop III stars; perhaps some even still exist to low redshifts.

\section{Later stages: Capture}

There is another possible source of DM in the first stars: capture of DM particles from the ambient medium. Whereas capture is negligible during the pre-mainsequence phase, once fusion sets in it can be important, depending on the value of the scattering cross section of DM with the gas. Two simultaneous papers $([6,7])$ found the same basic idea: the DM luminosity from captured WIMPs can be larger than fusion for the DS. Two uncertainties exist here: the scattering cross section, and the amount of DM in the ambient medium to capture from ${ }^{1}$. DS studies including capture have assumed the maximal scattering cross sections allowed by experimental bounds and ambient DM densities that are never depleted. With these assumptions, DS evolution models with DM heating after the onset of fusion have now been studied in several papers ([8, 9, 10]). We have been pursuing similar research with Alex Heger on DS evolution after the onset of fusion.

\section{Conclusion}

The line of research begun in Florence over two years ago is reaching a very fruitful stage of development. Dark matter can play a crucial role in the first stars. The first stars to form in the universe may be Dark Stars: powered by DM heating rather than by fusion. Our work indicates that they may be very large $\left(850 M_{\odot}\right.$ for $100 \mathrm{GeV}$ mass WIMPs). The connections between particle physics and astrophysics are ever growing!

\section{References}

[1] K. Freese, P. Bodenheimer, D. Spolyar, \& P. Gondolo 2008, arxiv:0806.0617 [astro-ph]

[2] P. Gondolo et al, these proceedings.

[3] A. Heger \& A. Woosley 2002, ApJ, 567, 532

[4] Y. X. Li et al. 2007, ApJ, 665, 187

[5] F. I. Pelupessy, T. Di Matteo and B. Ciardi 2007, arXiv:astro-ph/0703773.

[6] K. Freese, D. Spolyar, \& A. Aguirre 2008, arXiv:0802.1724 [astro-ph]

[7] F. Iocco 2008, ApJ Letters, 677, 1

\footnotetext{
${ }^{1}$ Unlike the annihilation cross section, which is set by the relic density, scattering is to some extent a free parameter set only by bounds from direct detection experiments.
} 
[8] F. Iocco, A. Bressan, E. Ripamonti, R Schneider, A. Ferrara \& P. Marigo 2008, arXiv:0805.4016

[9] M. Taoso, G. Bertone, G. Meynet \& S. Ekstrom 2008, arXiv:0806.2681 [astro-ph]

[10] S.Yoon, F. Iocco \& S. Akiyama 2008, arxiv:0806.2662 [astro-ph] 